\title{
Ginkgo Biloba Extract Protects Substantia Nigral Neurons from Apoptosis in PD Rat Model
}

Cao Fei*, Luo Fang, Zhang Lei, Su Ying, Chen Li, Hu Bo and Tong E-tang

Department of Neurology, Union Hospital, Tongji Medical College, Huazhong University of Science and Technology, Wuhan, China

\begin{abstract}
Purpose: To investigate the apoptotic mechanism during the pathological process of Parkinson disease (PD) and explore the availability of Ginkgo biloba extract (EGb) to cure PD.

Methods: Followed 6-OHDA injection stereotaxically to right side of substantia nigra pars compacta(SNc), at the date of 1, 7,14 and 21, we used biochemical, immunohistochemical, TUNEL and electron microscopic techniques to evaluate the level of apoptosis (TUNEL), monitor the degree of oxidative reaction (MDA.SOD), and to observe the change of ultrastructure of substantia nigra in the experimental PD models of rats (PD group), the EGb treatment group (EGb group) and the control group.
\end{abstract}

Results: Contrast to the Control group, the number of apoptotic neurons and free radical level increased in the PD group and the EGb group, but the PD group surpassed the EGb group $(p<0.05)$.In the PD group, the ultrastructure of substantia nigra exhibited the morphological characteristics of apoptosis from $1 \mathrm{~d}$ to $21 \mathrm{~d}$.

Conclusions: Free radical insult contributes to the pathological process of PD in rats. EGb may ameliorate the injury of substantia nigra through expiring free radicals during the pathological process of PD in rats.

Keywords: Ginkgo Biloba Extract (EGb); Parkinson disease; Apoptosis; Free radical

\section{Introduction}

Parkinson disease is one of the most common diseases in senile. Recent research point out that PD is an episode induced by multiple factors, such as oxidation stress and apoptosis. But the concrete mechanism still should be elucidated. 6-hydroxydopamine (6-OHDA), the dopamine metabolite which is involved in the pathological process of $\mathrm{PD}$, is often used as a chemical damage preparation to mimic dopaminergic nerve injury in vivo. Ginkgo Biloba extract (EGb), which was reported in Spirit of Ben Chao Ping Hui, was gymnosperm and has been used as drug for thousand of years in China. In this paper, we will explore the mechanism of EGb to protect the substantia nigra neurons and discuss the possibility of applying it to clinic work.

\section{Material and Methods}

All the following procedures have been approved by the Ethic Committee of Animal Care and Use of Tongji Medical College. Total 130 male Sprague Dawley rats (offered by Animal Center of Tongji Medical College), with body weight 180-200 g (aged 3 months), were used in this experiment.

\section{EGb suspension preparation}

EGb, with the commodity name of Tapning, was offered by Kang Engbei Medical Conglomerate of Zhejiang province. The component of EGb included $24 \%$ omni-flavone and $6 \%$ ginkgo lactone, which were solved in the $0.5 \%$ carboxymethyl cellulose sodium. Concentration of EGb suspension was $20 \mathrm{mg} / \mathrm{m}$.It should be used as soon as dispensed and preserved in hypothermia, avoiding of light and enclosed. Before being used, EGb suspension should be agitated by BIM-8A ultrasound shaker.

\section{PD rat models operation}

The PD rat models were operated according to the improved Thomas's method with little alterations [1]. Before 6-OHDA injection to the right substantia nigra, generally speaking, all rats were perfused consecutively through stomach with EGb suspension (Dosage was $100 \mathrm{mg} / \mathrm{kg} / \mathrm{d}$ ) for 7 days. After anesthesia with $10 \%$ chloral hydrate, the 6-OHDA was injected stereotaxically into the right substantia nigra. In the following days, the EGb group rats were undergone consecutively stomach perfusion with EGb suspension each day till the date point, while the Control group and the PD group were perfused with isometric normal saline.

\section{Tunel}

The apoptotic neurons were detected by the TUNEL method according to Seaton's method $[2,3]$. After anesthesia with $10 \%$ chloral hydrate, the rats were sacrificed. Then the midbrain was taken out and made into frozen preparation sections. Through HE staining, the sections with clear-cut damage of substantia nigra were reserved at $4^{\circ} \mathrm{C}$ and used in the followed procedures. Then the apoptotic neurons were examined by TUNEL test kit according to the manufacturer provided by Nanking Jiancheng Corporation, China, and the apoptotic cells were counted.

\section{Lipid peroxidate products and free radical expiring ability examination}

After anesthesia with $10 \%$ chloral hydrate, the rats were sacrificed. Then the tissue of right substantia nigra was fetched. Lipid peroxidate products and free radical expiring ability were examined by malondialdehyde (MDA) and superoxide dismutase (SOD) biological

*Corresponding author: Cao Fei, Department of Neurology, Union Hospital, Tongji Medical College, Huazhong University of Science and Technology, Wuhan, China, E-mail: caofyx@163.com

Received April 11, 2013; Accepted May 27, 2013; Published May 29, 2013

Citation: Fei C, Fang L, Lei Z, Ying S, Li C, et al. (2013) Ginkgo Biloba Extract Protects Substantia Nigral Neurons from Apoptosis in PD Rat Model. J Neurol Disord 1: 118. doi:10.4172/2329-6895.1000118

Copyright: (C) 2013 Fei C, et al. This is an open-access article distributed under the terms of the Creative Commons Attribution License, which permits unrestricted use, distribution, and reproduction in any medium, provided the original author and source are credited. 
product test kit according to the manufacturer provided by Nanking Jiancheng Corporation, China.

\section{Observation of electron microscope}

$0.5 \mathrm{~mm}^{3}$ substantia nigra was picked out and double fixed with $2.5 \%$ glutaral, $1 \%$ osmium acid lead, and then desiccation and embed in Epon812. Ultrathin sections were made by LKB-III microtome, double stained by acetic acid $U$ and lead nitrate, and then observed by transmission electron microscope.

\section{Statistical analysis}

All data were presented as mean \pm standard deviation (SD), and analyzed by one-way ANOVA. A value of $p<0.05$ was taken as significant.

\section{Results}

\section{Counting apoptotic cells of substantia nigra}

The number of apoptotic cells in the PD group and EGb group was increased gradually compared with the Control group, but the EGb group was fewer than the PD group; Compared with the PD group, the EGb group decreased 57\%,54\%, 48\% and 50\% respectively at each time point (Table 1).

\section{SOD and MDA in substantia nigra}

There was no notable change of MDA and SOD level in the Control group at each time point. Contrast to the Control group, both of MDA and SOD in the PD and the EGb group was increasing with time, but the level of MDA and SOD in the EGb group was less than that of the PD group (Tables 2 and 3 ).

\section{Electron microscopic observation of the ultrastructure of substantia nigra}

1d PD group: The cellular membrane of substantia nigra neurons presented "blister phenomenon". Nuclear membrane was irregular, and cytoplasm was concentrated.

\begin{tabular}{|l|l|l|l|l|l|}
\hline Groups & $\mathbf{n}$ & \multicolumn{4}{|l|}{ Days after 6-OHDA injection } \\
\cline { 3 - 6 } & & $1 \mathrm{~d}$ & $7 \mathrm{~d}$ & $14 \mathrm{~d}$ & $21 \mathrm{~d}$ \\
\hline Control & 20 & $65 \pm 8$ & $55 \pm 9$ & $71 \pm 11$ & $56 \pm 8$ \\
\hline PD & 20 & $358 \pm 31^{*}$ & $388 \pm 48^{*}$ & $413 \pm 22^{*}$ & $456 \pm 14^{*}$ \\
\hline EGb & 20 & $154 \pm 18^{\star} \#$ & $179 \pm 21^{\star} \#$ & $212 \pm 21^{\star} \#$ & $228 \pm 28^{\star} \#$ \\
\hline
\end{tabular}

${ }^{*} p<0.05$ vs. Control group, $\# p<0.05$ vs. EGb group

Table 1: Number of apoptotic cells of substantia nigra $\left(/ \mathrm{mm}^{2}\right)$.

\begin{tabular}{|l|l|l|l|l|l|}
\hline Groups & n & \multicolumn{5}{|l|}{ Days after 6-OHDA injection } \\
\cline { 3 - 6 } & & $1 \mathrm{~d}$ & $7 \mathrm{~d}$ & $14 \mathrm{~d}$ & $21 \mathrm{~d}$ \\
\hline Control & 20 & $107 \pm 18$ & $109 \pm 17$ & $113 \pm 11$ & $104 \pm 17$ \\
\hline PD & 20 & $135 \pm 11^{*}$ & $165 \pm 15^{*}$ & $181 \pm 20^{*}$ & $197 \pm 22^{*}$ \\
\hline EGb & 20 & $104 \pm 19^{\star} \#$ & $112 \pm 20^{*} \#$ & $137 \pm 19^{*} \#$ & $148 \pm 12^{*} \#$ \\
\hline
\end{tabular}

${ }^{*} p<0.05$ vs. control group, \#p<0.05 vs. EGb group

Table 2: MDA in substantia nigra (NU/mg).

\begin{tabular}{|l|l|l|l|l|l|}
\hline Groups & $\mathbf{n}$ & \multicolumn{4}{|l|}{ Days after 6-OHDA injection } \\
\cline { 3 - 6 } & & $1 \mathrm{~d}$ & $7 \mathrm{~d}$ & $14 \mathrm{~d}$ & $21 \mathrm{~d}$ \\
\hline Control & 20 & $350 \pm 36$ & $355 \pm 27$ & $357 \pm 25$ & $362 \pm 30$ \\
\hline PD & 20 & $398 \pm 31^{*}$ & $409 \pm 39^{*}$ & $435 \pm 29^{*}$ & $472 \pm 41^{*}$ \\
\hline EGb & 20 & $227 \pm 30^{*} \#$ & $258 \pm 33^{*} \#$ & $265 \pm 32^{*} \#$ & $288 \pm 36^{*} \#$ \\
\hline
\end{tabular}

${ }^{*} p<0.05$ vs. Control group, $\# p<0.05$ vs. EGb group

Table 3: SOD in substantia nigra $(\mathrm{nmol} / \mathrm{g})$. 1d EGb group: The shape of substantia nigra neurons was normal, and the nuclear membrane was regular.

7d PD group: Neurons of substantia nigra were diminished. Concentrated chromatins were encircled around the edge of the nuclei like a crescent.

$7 \mathrm{~d}$ EGb group: The concentrated chromatins were encircled at the edge of the nucleus, and the nuclear membrane became irregular.

14d PD group: Neurons of substantia nigra were diminished. Concentrated chromatin presented like a mass and the glial cells were proliferated.

14d EGb group: The nuclei of substantia nigra neurons were concentrated, and divided into several leaves with focal edema.

21d PD group: Neurons of substantia nigra were shrunken. Nuclei were concentrated into a mass and the Satellitosis phenomenon could be seen. Moreover, the apoptotic neurons were phagocyted.

21d EGb group: Neurons of substantia nigra were shrunken. Concentrated chromatins were encircled at the edge of nucleus. Phenomenon of blister could be seen.

\section{Discussion}

6-OHDA can lead to oxidative stress injury and produce free radicals, which reflected the degree of injury of cells indirectly $[3,4]$. While the vitality of SOD reflected the ability of body to clean off free radicals, and SOD vitality was increased in the experimental models, the increased SOD level cannot compensate the injury. Choi [5] thought that the degree of SOD increasing is less than that of free radical increasing by far in PD patients. In our study, we found that the level of MDA and vitality of SOD increased gradually with the injury time. This effect revealed that the oxidative stress injury of substantia nigra was getting even more serious gradually.

Apoptosis was proved to be the common pathway in the episode of PD. Recent study in vitro found that free radicals played a major role on cell apoptosis [6]. In our study, we found that the number of apoptotic cells of substantia nigra was increased gradually from $1 \mathrm{~d}$ to $21 \mathrm{~d}$, and the ultrastructure of substantia nigra presented typical apoptosis alteration.

As a classic agent of Chinese traditional medicine, EGb can clean off free radicals. Some literature reported that EGb can prevent the granular cells of cerebellum, hippocampus neurons of rats from apoptosis $[7,8]$. In our study, we found that the level of MDA and SOD in the EGb group was less than the PD group notably, which mean oxidative stress injury in the EGb group was less serious than the PD group. Our study also found that EGb can prevent the cells of substantia nigra from apoptosis effectively. The apoptotic cells of substantia nigra in the EGb group decreased 57\%, 54\% 48\%, 50\% compared with the PD group at the date of $1,7,14,21$ respectively. The ultrastructural damage of the EGb group was less than the PD group either. EGb can protect neurons in many ways because of its multi-component and multi-valence pharmacologic effects. EGb can decrease not only membrane fluidity and protein conformation change evoked by free radicals, but also the concentration of peroxide anion, for instance, it can conjugate with peroxidase prosthetic group $\mathrm{Fe}^{3+}$ and relieve neuronal toxic effect of glutamate acid [9]. More over, it can reduce toxic effect of MPTP and prevent the injury of mitochondria and DNA.

Taking together, our study found that EGb protect neurons from 
Citation: Fei C, Fang L, Lei Z, Ying S, Li C, et al. (2013) Ginkgo Biloba Extract Protects Substantia Nigral Neurons from Apoptosis in PD Rat Model. J Neurol Disord 1: 118. doi:10.4172/2329-6895.1000118

Page 3 of 3

free radicals and decreased the apoptosis ratio and the ultrastructure damage of substantia nigra in PD rat model. EGb might alleviate the symptom of PD and become a potential drug for PD patients.

\section{References}

1. Thomas J, Wang J, Takubo H, Sheng J, de Jesus S, et al. (1994) A 6-hydroxydopamine-induced selective parkinsonian rat model: further biochemical and behavioral characterization. Exp Neurol 126: 159-167.

2. Seaton TA, Cooper JM, Schapira AH (1998) Cyclosporin inhibition of apoptosis induced by mitochondrial complex I toxins. Brain Res 809: 12-17.

3. Tompkins MM, Basgall EJ, Zamrini E, Hill WD (1997) Apoptotic-like changes in Lewy-body-associated disorders and normal aging in substantia nigral neurons. Am J Pathol 150: 119-131.

4. Camp DM, Loeffler DA, LeWitt PA (2000) L-DOPA does not enhance hydroxyl radical formation in the nigrostriatal dopamine system of rats with a unilateral 6-hydroxydopamine lesion. J Neurochem 74: 1229-1240.
5. Choi WS, Yoon SY, Oh TH, Choi EJ, O'Malley KL, et al. (1999) Two distinct mechanisms are involved in 6-hydroxydopamine- and MPP+-induced dopaminergic neuronal cell death: role of caspases, ROS, and JNK. J Neurosci Res 57: 86-94.

6. France-Lanord V, Brugg B, Michel PP, Agid Y, Ruberg M (1997) Mitochondria free radical signal in ceramide-dependent apoptosis: a putative mechanism for neuronal death in Parkinson's disease. J Neurochem 69: 1612-1621.

7. Wei T, Ni Y, Hou J, Chen C, Zhao B, et al. (2000) Hydrogen peroxide-induced oxidative damage and apoptosis in cerebellar granule cells: protection by Ginkgo biloba extract. Pharmacol Res 41: 427-433.

8. Ahlemeyer B, Möwes A, Krieglstein J (1999) Inhibition of serum deprivationand staurosporine-induced neuronal apoptosis by Ginkgo biloba extract and some of its constituents. Eur J Pharmacol 367: 423-430.

9. Zhou LJ, Zhu XZ (2000) Reactive oxygen species-induced apoptosis in PC12 cells and protective effect of bilobalide. J Pharmacol Exp Ther 293 982-988 